\title{
Cylindrical wormholes in DGP gravity
}

\author{
Martín G. Richarte1, 回 \\ 1 Departamento de Fúsica, Facultad de Ciencias Exactas y Naturales, \\ Universidad de Buenos Aires and IFIBA, CONICET, \\ Ciudad Universitaria, Pabellón I, 1428, Buenos Aires, Argentina
}

\begin{abstract}
We construct traversable thin-shell wormholes in the Dvali-Gabadadze-Porrati theory with cylindrical symmetry applying the cut and paste procedure to a flat black string solution of the fivedimensional vacuum Einstein field equations. In contrast to general relativity case, where thin-shell wormholes violate both weak and null energy conditions, we show that static wormholes are supported by normal matter while vacuum wormholes do not exist.
\end{abstract}

PACS numbers:

Keywords:

\section{INTRODUCTION}

Alternative gravity theories such as one proposed by Dvali, Gabadadze, and Porrati (DGP) claims that our observed four-dimensional universe lives in a larger fivedimensional space-time. In fact, gravity is modified at large (rather than at short) distances through the slow evaporation of gravitational degrees of freedom of the brane universe [1]. The transition between four and higher-dimensional gravitational potentials in the DGP model arises because of the presence of both the brane and bulk Hilbert-Einstein terms in the action 11. Despite the brane universe rendering the cosmic acceleration without need of any vacuum (dark energy) term [2], it suffers from some instabilities due to the existence of ghost-like excitations [3].

Any attempt to construct thin-shell wormholes requires the use of the cut and paste procedure [4], [5] and work with the junction conditions associated to the gravity theory understudy [6]. Cylindrical thin shell wormholes within the context of general relativity (GR) were built, and found that, in most of the cases, the wormholes are supported by exotic matter, violating the energy conditions [7]. Leaving aside the GR by taking into account the Brans-Dicke gravity theory, it was shown that cylindrical thin-shells are not necessarily are sourced by exotic matter and the energy conditions can be fulfilled by choosing suitably the parameters of the model [8].

In the case of DGP-gravity theory, spherically symmetric thin-shell wormholes have been constructed by gluing two copies of the same five-dimensional vacuum solution (Schwarzschild black hole), where the throat of the wormhole is located at the joining surface [9]. It turns out that the extra Hilbert-Einstein term at the boundary has meaningful effects by generalizing the junction conditions [10]; thereby it incorporates the Einstein tensor in the projected field equations on the joining surface, altering substantially the kind of matter which could sup-

${ }^{\text {a} E l e c t r o n i c ~ a d d r e s s: ~ m a r t i n @ d f . u b a . a r ~}$ port wormhole configurations. The contributions form the curvature tensor, theoretically, and seem to allow the existence of wormholes supported by ordinary matter as well as solitonic solutions, that is, vacuum shells which are gravitationally self supported due to the nonlinear character of the junction conditions 9 ].

In the present work, we started by implementing the cut and paste procedure on the black string solution in order to construct static thin-shell wormholes with cylindrical symmetry, and then, we employed the generalized junction conditions within the DGP gravity to determine the energy density and pressure on the shell. We are going to address a key issue regarding the kind of matter which could support these wormholes configurations. We would like to know whether the DGP gravity provides a suitable framework to allow the existence of cylindrical wormholes which do not violate the energy conditions. In the next sections, without loss of generality, we will work with units such that $8 M_{5}^{3}=1$.

\section{THE DGP GRAVITY}

We begin by setting out the action corresponding to the DGP theory in a five-dimensional manifold $\mathcal{M}_{5}$ with a four-dimensional boundary $\Sigma$ (cf. [10]),

$$
\begin{aligned}
S & =2 M_{5}^{3} \int_{\mathcal{M}_{5}} d^{5} x \sqrt{-g} R\left[g_{A B}\right]+2 M_{4}^{2} \int_{\Sigma} d^{4} x \sqrt{-\gamma} \mathcal{R}\left[\gamma_{a b}\right] \\
& +4 M_{5}^{3} \int_{\Sigma} d^{4} x \sqrt{-\gamma}\left(-\mathcal{K}\left[\gamma_{a b}\right]+\frac{\mathcal{L}_{m}}{4 M_{5}^{3}}\right),
\end{aligned}
$$

where $g_{A B}$ is the five-dimensional metric, $\gamma_{a b}$ is the fourdimensional induced metric on the boundary $\Sigma$, and $\mathcal{K}$ is the trace of extrinsic curvature. Here, the matter fields in $\mathcal{L}_{m}$ are confined to a 4 -dimensional boundary. The extra term in the boundary introduces a mass scale $m_{c}=2 M_{5}^{3} / M_{4}^{2}=r_{c}^{-1}$ which determines a scale that separates two different regimes of the theory. For distances much smaller than $m_{c}^{-1}$ one would expect the solutions to be well approximated by general relativity and the modifications to appear at larger distances. This is 
indeed the case for distributions of matter and radiation which are homogeneous and isotropic at scales $\gtrsim r_{c}$. Typically, $m_{c} \simeq 10.42 \mathrm{GeV}$, so it sets the distance/time scale $r_{c}=m_{c}^{-1}$ at which the Newtonian potential significantly deviates from the conventional one. In the bulk the DGP equations are the Einstein ones in vacuum : $G_{A B}^{(5)}=0$. For the coordinate $X^{A}=(t, r, \theta, \phi, y)$ the bulk metric corresponding to cylindrical black hole vacuum solution takes the form

$$
\begin{aligned}
g_{A B} & =\operatorname{diag}\left(-f(r),[f(r)]^{-1}, r^{2}, r^{2} \sin ^{2} \theta, 1\right), \\
f(r) & =\left(1-\frac{r_{+}}{r}\right),
\end{aligned}
$$

where the parameter $m$ is related to the Arnowitt-DeserMisner mass. The above space-time has only one horizon placed at $r_{+}=2 m$ with $m>0$. Besides, when $m<0$, the manifold only presents a naked singularity at the origin $r=0$ which can be easily verified through the squared Riemann tensor, given by $R_{A B C D} R^{A B C D}=10 r^{2}+/ r^{6}$ [11].

\section{THIN-SHELL CONSTRUCTION IN DGP THEORY}

Employing the metric Eqs.(1-2), we build a spherically symmetric thin-shell wormhole in DGP theory. We take two copies of the space-time and remove from each manifold the five-dimensional regions described by

$$
\mathcal{M}_{ \pm}=\left\{x / r_{ \pm} \leq a, a>r_{+}\right\} .
$$

where $a$ is chosen to include possible singularities or horizon within the region $\mathcal{M}_{ \pm}$. The resulting manifolds have boundaries given by the timelike hypersurfaces,

$$
\Sigma_{ \pm}=\left\{x / r_{ \pm}=a, a>r_{+}\right\} .
$$

Then we identify these two timelike hypersurfaces to obtain a geodesically complete new manifold $\mathcal{M}=\mathcal{M}^{+} \cup$ $\mathcal{M}^{-}$with a matter shell at the surface $r=a$ where the throat of the wormhole is located. This manifold is constituted by two regions which are asymptotically flat. To study this type of wormholes we apply the Darmois-Israel formalism generalized [10] to the case of the DGP theory. We can introduce the coordinates $\xi^{a}=(\tau, \theta, \phi, y)$ in $\Sigma$, with $\tau$ the proper time on the throat. We will focus in static configurations, then the boundary hypersurface reads:

$$
\Sigma: \mathcal{H}(r)=r-a=0
$$

The field equations projected on the shell $\Sigma$ are the generalized junction (or Darmois-Israel) conditions [10],

$$
r_{c}\left[\mathcal{R}_{a b}-\frac{1}{2} \gamma_{a b} \mathcal{R}\right]-2\left\langle\mathcal{K}_{a b}-\gamma_{a b} \mathcal{K}\right\rangle=\mathcal{S}_{a b},
$$

where the bracket $\langle$.$\rangle stands for the jump of a given quan-$ tity across the hypersurface $\Sigma$ and $\gamma_{a b}$ is the induced metric on $\Sigma$. The extrinsic curvature $\mathcal{K}_{a b}$ is defined as follows:

$$
\mathcal{K}_{a b}^{ \pm}=-n_{A}^{ \pm}\left(\frac{\partial^{2} X^{A}}{\partial \xi^{a} \partial \xi^{b}}+\Gamma_{B C}^{A} \frac{\partial X^{B}}{\partial \xi^{a}} \frac{\partial X^{C}}{\partial \xi^{b}}\right)_{r=a},
$$

where $n_{A}^{ \pm}$are the unit normals to the surface $\Sigma$.

Notice that the first term in (6) is not enclosed within the brackets because this contribution comes from the four-dimensional Hilbert-Einstein term in the DGP action which already lives in the boundary, so it does not need to be projected on $\Sigma$. By taking the limit $r_{c} \rightarrow 0$, we recover the standard Darmois-Israel junction condition found in [6]. In order to proceed one can write the intrinsic metric to $\Sigma$ as

$$
d s_{\Sigma}^{2}=-d \tau^{2}+a^{2}\left(d \theta^{2}+\sin ^{2} \theta d \phi^{2}\right)+d y^{2} .
$$

The position of the junction surface is given $X^{A}=$ $(t(\tau), a, \theta, \phi, y)$ and the corresponding 4 velocity is $u^{A}=$ $\left([f(a)]^{-1 / 2}, 0,0,0,0\right)$, whereas the unit normal to shell may be determine by the conditions $u^{A} n_{A}=0$ and $n^{B} n_{B}=1$. These requisites lead to the following expression, $n_{A}=\left(0,[f(a)]^{-1 / 2}, 0,0,0\right)$. Noncompact wormhole geometries such as the one described by Eq. (8) admit different variants of the definition of a throat [12]. For instance, the volume per unit length is given by $\mathcal{V}(a) / \ell=4 \pi a^{2}$ and is an increasing function on both side of the throat. Notice that the throat has translational symmetry along the $y$ direction, but when one considers $y$ fixed, the global properties are determined by the behavior of topology of the throat. So our example corresponds to a sphere with area $\mathcal{A}(a)=4 \pi a^{2}$, thus it has a minimal "area" surface reaching a minimum at the position of the throat. Now, if we look at the topology of the throat for $y$ and $\phi$ fixed, we find that the circular radius function $\mathcal{R}(a)=2 \pi a$ defines its perimeter and it can be considered as a less restrictive definition of the wormhole throat 12.

Now, let us calculate some quantities that we will need later. The mixed components of the four-dimensional Einstein tensor are given by

$$
\begin{aligned}
& \mathcal{G}^{0}{ }_{0}=-\frac{1}{a^{2}}=\mathcal{G}^{y}, \\
& \mathcal{G}^{\theta}{ }_{\theta}==\mathcal{G}^{\phi}{ }_{\phi}=0 .
\end{aligned}
$$

The extrinsic curvature components read

$$
\begin{aligned}
\left\langle\mathcal{K}_{0}^{0}\right\rangle & =\frac{f^{\prime}(a)}{\sqrt{f(a)}},\left\langle\mathcal{K}^{y}{ }_{y}\right\rangle=0 . \\
\left\langle\mathcal{K}^{\theta}{ }_{\theta}\right\rangle & =\frac{2}{a} \sqrt{f(a)}=\left\langle\mathcal{K}^{\phi}{ }_{\phi}\right\rangle .
\end{aligned}
$$

The component $\mathcal{K}_{y}^{y}$ of the extrinsic curvature vanishes because the fifth dimension is flat, that is, this component involves the metric and its derivatives while $\mathcal{G}^{y}{ }_{y}$ is 
nonzero because has a term proportional to the fourdimensional Ricci scalar $\mathcal{R}$.

The most general form of the stress energy tensor on shell compatible with the space-time symmetries is

$$
\mathcal{S}^{a}{ }_{b}=\operatorname{diag}\left(-\sigma, P_{\theta}, P_{\theta}, P_{y}\right)
$$

After some algebraic manipulation, we obtain that the energy density and the tangential pressures can be recast as

$$
\begin{array}{r}
\sigma=\frac{r_{c}}{a^{2}}-\frac{8 \sqrt{f(a)}}{a}, \\
P_{\theta}=2 \frac{f^{\prime}(a)}{\sqrt{f(a)}}+\frac{4 \sqrt{f(a)}}{a}, \\
P_{y}=-\frac{r_{c}}{a^{2}}+\frac{2 f^{\prime}(a)}{\sqrt{f(a)}}+\frac{8 \sqrt{f(a)}}{a} .
\end{array}
$$

The DGP contributions are encoded in the $r_{c}$ factor of the above equations.

\section{MATTER SUPPORTING THE WORMHOLES}

Classical solutions within the DGP model corresponding to thin-shell wormholes were found in [9], where the stability analysis indicated that these configurations could be stable, moreover the matter supporting them can be interpreted as nonrelativistic in some cases due to their very small squared speed sound [9]. All these elements seem to be good reasons to consider a careful discussion about the nature of matter supporting wormholes with cylindrical symmetry within the DGP model. Following the approach presented in [9] where the four-dimensional Hilbert-Einstein generalizes the standard junction, adding the Einstein tensor on the shell and due to its geometrical nature, the next approach will clearly be the most suitable framework to give a precise meaning to the characterization of matter supporting the wormhole with cylindrical symmetry.

The weak energy condition (WEC) states that for any timelike vector $u^{A}$ it must be $T_{A B} u^{A} u^{B} \geq 0$; the WEC also implies, by continuity, the null energy condition (NEC), which means that for any null vector $k^{A}$ it must be $T_{A B} k^{A} k^{B} \geq 0$ [4]. In an orthonormal basis the WEC reads $\rho \geq 0, \rho+P_{l} \geq 0 \forall l$ while the NEC takes the form $\rho+P_{l} \geq 0 \forall l$.

In the case of thin-shell wormholes the radial pressure $P_{r}$ is zero, and within Einstein gravity, the surface energy density must fulfill $\sigma<0$ so that both energy conditions will be violated. The sign of $\sigma+P_{t}$, where $P_{t}$ is the transverse pressure is not fixed, but it depends on the values of the parameters of the system.

Now, the sign of the surface energy density (14) as well as the pressure along axis $y(16)$ is, in principle, not fixed. For $r_{c} \rightarrow 0$, we obtain the energy density and pressures for cylindrical wormhole geometries as if it was calculated with the standard junction conditions. Far away from the general relativity limit we now find that there exist positive contributions to $\sigma$. We stress that this would not be possible if the standard Darmois-Israel formalism was applied; treating the DGP contribution as an effective energy-momentum tensor, thereupon we inevitably would obtain that the energy density is negative definite because the flare-out condition is fulfilled, so this leads to $\sigma=-8\left[f(a) / a^{2}\right]^{1 / 2}<0$.

Now, once the explicit form of the function $f(a)$ is introduced in Eq.(14), we focus on the conditions that lead to wormholes with $\sigma>0$. Then, it can be proven that wormholes with a non-negative surface density located at the shell are allowable when the following inequalities are simultaneously satisfy:

$$
\frac{r_{c}}{a^{2}}-\frac{8}{a}\left(1-\frac{r_{+}}{a}\right)^{\frac{1}{2}}>0 \cap a-r_{+}>0,
$$

so it is always possible to choose $a$ such that the existence of thin-shell wormholes is compatible with positive surface energy density. More precisely. its radius must belong to the interval given below,

$$
r_{+}<a \leq \frac{r_{+}}{2}+\frac{1}{2}\left(r_{+}^{2}+\frac{r_{c}^{2}}{16}\right)^{\frac{1}{2}}
$$

Notice that the $r_{c}$-term is essential to have positive energy density; as one would expect, in the limit $r_{c} \longrightarrow 0$, this possibility completely vanishes. Besides, the sum of the pressure $P_{\theta}$ and energy density $\sigma$ takes the form

$$
\sigma+P_{\theta}=\left(\frac{r_{c}}{a^{2}}+\frac{2 a f^{\prime}(a)-4 f(a)}{a \sqrt{f(a)}}\right),
$$

because the first term in (19) is positive the sign of $\sigma+P_{\theta}$ depends on the second term, implying that the sum is positive for $r_{+}<a \leq 3 r_{+} / 2$. Therefore, the remarkable result is that, now we have a region with $\sigma \geq 0$ along with $\sigma+P_{\theta} \geq 0$. In addition, $\sigma+P_{y}=2 f^{\prime}(a) / \sqrt{f(a)}$ is always positive, showing that wormholes with cylindrical symmetry within the DGP framework satisfy both WEC and NEC.

We have obtained a nontrivial inequality (19) about the range that wormhole's radius must cover in order to get positive energy density. As is well known a physical wormhole solution is interestingly enough as long as the wormhole's radius it is not restricted to a small region in the parameter space defined by $r_{c}$ and $r_{+}$. Therefore, it is important to check the nonexistence of such fine-tuning between these two parameters, hence such analysis implies to know the order of magnitude of $r_{c} / r_{+}=4 \pi r_{c} M_{4}^{2} / m$, where the four-dimensional Newton constant is $G_{4}=1 / 8 \pi M_{4}^{2}$. The cosmological estimation of the crossover scale using supernovae type Ia data leads to $r_{c}=5 \mathrm{Gpc}$, whereas the parameter $m$ can be taken as the black hole's mass. One could infer the numerical value of $r_{c} / r_{+}=(1.33) \eta^{-1} \times 10^{24}$ by written the black hole mass in terms of the sun's mass provided $m=\eta M_{\odot}$, where $\eta$ encodes the size of black hole. 
For black holes that are formed in the collapse of massive stars with stellar-mass $(\eta \simeq 10)$ imply that the ratio $r_{c} / r_{+} \simeq \mathcal{O}\left(10^{23}\right)$, whereas for supermassive black holes that reside in galactic centers, this is tantamount to saying that $10^{6} \leq \eta \leq 10^{10}$ [13]; the ratio covers interval $\left[10^{14} ; 10^{18}\right]$. For instance, studying the dynamics of gas in the early-type galaxy NGC 4526, the statistical analysis leads to a central dark object of $4.5 \times 10^{8} M_{\odot}$ at $3 \sigma$ confidence level [14]. In this case, one obtains that $r_{c} / r_{+} \simeq 2.9 \times 10^{15}$. Another appealing case seems to be that of black holes much less massive than a solar mass $M_{\odot}$ possibly formed as result of density perturbations in the early Universe. In the latter cases, the ratio is considerably amplified by many orders of magnitude provided that one always has $\eta \leq 10^{-1}$. In all the cases mentioned before, we are able to ensure that $r_{c} / r_{+}$is large enough to avoid any kinds of fine-tunings.

Finally, we would like to show that there are not vacuum wormholes with cylindrical symmetry selfgravitating due to the nonlinearity of the junction conditions within the framework of DGP gravity. In order to show that, we must impose that energy density and tangential pressures vanish at the same time. As it can be seen from Eq. (14) and Eq.(16), one can attain that $\sigma$ and $P_{y}$ vanish for a suitably radius, but Eq. (15) tells us that $P_{\theta}$ cannot become null; such a finding does not occur in the case of spherically symmetric wormholes [9].

\section{SUMMARY AND DISCUSSION}

In this work, we have taken a vacuum black string solution of the five-dimensional Einstein field equations and followed the cut and paste method for removing the singular part of this manifold in order to construct a cylindrical wormhole. We have shown that wormholes with cylindrical symmetry within the framework of the DGP gravity theory do exist due to the nonlinear corrections that the boundary Hilbert-Einstein term adds to the generalized junction conditions.

We have also proven that in a possible scenario, where the DGP crossover scale $r_{c}$ is considerably large enough in relation with the horizon radius $r_{+}$, corresponding to a situation far away from the general relativity limit, the energy density located at the wormhole's throat can be positive; moreover, it turned out that such gravitating configurations do fulfill the weak and null energy conditions. We have found that the wormhole's radius is not restricted to a small region in the parameter space avoiding any kind of fine-tuning that could exist; thus, the DGP gravity theory introduces a new parameter, which allows for more freedom in the framework of determining the most viable wormhole configurations without to be threatened by the presence of exotic matter. For stellar black holes with mass $m=10 M_{\odot}$, the ratio is $r_{c} / r_{+} \simeq$ $\mathcal{O}\left(10^{23}\right)$, whereas for supermassive black holes that reside in galactic centers with $10^{6} M_{\odot} \leq m \leq 10^{10} M_{\odot}$ [13], the ratio covers interval $\left[10^{14} ; 10^{18}\right]$. For instance, the statistical analysis performed to the early-type galaxy NGC 4526 leads to a central dark object of $4.5 \times 10^{8} M_{\odot}$ at $3 \sigma$ confidence level [14], implying that $r_{c} / r_{+} \simeq 2.9 \times 10^{15}$. Besides, one difference in relation to the case with spherical symmetry is that now we cannot construct static vacuum wormholes; thus, we cannot have self-gravitating solutions where both pressures and energy density vanish at the same time. Indeed, such impossibility arises because the anisotropic pressure along the axis where the black string is oriented in the original manifold does not vanish, however, we cannot discard that nonstatic vacuum wormholes do exist.

At this point, one issue that remains open and requires further investigation is concerned with the dynamic of cylindrical wormholes in the DGP theory (how these wormholes evolve when they are perturbated without altering their cylindrical symmetry). In fact, because the black string background does not have the same horizon topology as the five-dimensional schwarzschild solution. There is not a Birkhoff 's theorem that guarantees no emission of gravitational waves (radiation), and hence the geometry outside the throat could not remain static [11]. This fact indicates that a more careful analysis must be carried out in treating cylindrical perturbations for wormhole geometries.

\section{Acknowledgments}

We are grateful with the referee for his valuable comments that helped improve the article.

The author is partially supported by Postdoctoral Fellowship Programme of Consejo de Investigaciones Científicas y Técnicas (CONICET).
[1] G.R. Dvali, G. Gabadadze, and M. Porrati, Phys.Lett.B 485 208-214 (2000).; G. R. Dvali and G. Gabadadze, Phys. Rev. D 63, 065007 (2001) .

[2] C. Deffayet, Phys. Lett. B 502, 199 (2001);C. Deffayet, G. R. Dvali and G. Gabadadze, Phys. Rev. D 65, 044023 (2002)

[3] D. Gorbunov, K. Koyama, and S. Sibiryakov, Phys. Rev. D 73044016 (2006).

[4] M. Visser, Lorentzian Wormholes (AIP Press, New York,
1996).

[5] Francisco S.N. Lobo, Paulo Crawford, Class.Quant.Grav. 21 (2004) 391-404; E. F. Eiroa, G.E. Romero, Gen.Rel.Grav. 36 (2004) 651-659; J. P.S. Lemos and F. S.N. Lobo, Phys.Rev. D 69 (2004) 104007; M. G. Richarte and C. Simeone , Int.J.Mod.Phys.D 17:11791196,2008 M. G. Richarte and C. Simeone, Phys. Rev. D 76, 087502 (2007); Erratum-ibid 77, 089903 (2008); M. G. Richarte and C. Simeone, Phys.Rev. D 80 (2009) 
104033, Erratum-ibid. D 81 (2010) 109903; E. F. Eiroa, M. G. Richarte, and C. Simeone, Phys.Lett.A 373 14 (2008), Erratum-ibid.373:2399-2400, 2009; Goncalo A.S. Dias and Jose P.S. Lemos, Phys.Rev. D 82 (2010) 084023. S.Habib Mazharimousavi, M. Halilsoy, Z. Amirabi, Phys.Rev. D 81 (2010) 104002; M.H. Dehghani, M.R. Mehdizadeh, Phys.Rev. D 85 (2012) 024024; P. Kanti, B. Kleihaus, J. Kunz, Phys.Rev. D 85 (2012) 044007; M. G. Richarte, C. Simeone, Int.J.Mod.Phys.D 17 1179-1196 (2008); Ivana Bochicchio, Ettore Laserra, [arXiv:1301.153]; Farook Rahaman, A. Banerjee, I. Radinschi, Int.J.Theor.Phys. 51 (2012) 1680-1691; A.A. Usmani, Z. Hasan, F. Rahaman, Sk.A. Rakib, Saibal Ray, Peter K.F. Kuhfittig, Gen.Rel.Grav. 42 (2010) 29012912; Jose P.S. Lemos , Francisco S.N. Lobo, Phys.Rev. D 78 (2008) 044030; K. A. Bronnikov, A. A. Starobinsky, Mod.Phys.Lett. A24 (2009) 1559-1564.

[6] G. Darmois, Mémorial des Sciences Mathématiques, Fascicule XXV ch V (Gauthier-Villars, Paris, 1927) ; W. Israel, Nuovo Cimento 44B, 1 (1966); 48B, 463(E) (1967).
[7] E. F. Eiroa, C. Simeone, Phys.Rev. D 70 (2004) 044008; E. F. Eiroa , C. Simeone, Phys.Rev. D 81 (2010) 084022; M. G. Richarte, C.Simeone, Phys.Rev. D 79 (2009) 127502. C.Simeone, Int.J.Mod.Phys. D 21 (2012) 1250015 .

[8] E. F. Eiroa, C. Simeone, Phys.Rev. D 82 (2010) 084039.

[9] M. G. Richarte, Phys.Rev.D 82044021 (2010).

[10] R. Gregory, N. Kaloper, R. Myers, and A. Padilla, JHEP 0710069 (2007).

[11] R.Gregory and R. Laflamme, Phys.Rev. D37 (1988) 305308.

[12] K.A. Bronnikov and J.P.S. Lemos, Phys. Rev. D 79, 104019 (2009).

[13] M. Kesden and S. Hanasoge, PRL 107, 111101 (2011)

[14] A black-hole mass measurement from molecular gas kinematics in NGC4526 T. A. Davis, M. Bureau, M. Cappellari, M. Sarzi, L. Blitz, Nature 494, 328330 (2013). doi:10.1038/nature11819. 